\title{
RECHERCHES SUR LA BIOCHIMIE DES COMPOSÉS PHOSPHORÉS DU LAIT (1)
}

par

\section{H. SIMONNET et J. STERNBERG}

L'étude de l'élaboration de la caséine comporte deux aspects fondamentaux: l'un extra-mammaire : origine de ses constituants azotés et phosphorés et de la forme sous laquelle ils sont apportés à la glande, l'autre intra-mammaire : mécanisme d'élaboration et de sécrétion de la micelle de caséine.

En ce qui concerne l'origine du matériel azoté, les recherches de TURNER et ses collaborateurs [1] ont abouti à la conclusion qu'un glycoprotéide sanguin est à l'origine de la fraction amino-acidique. Quant à la source du matériel phosphoré, des origines diverses ont été proposées, sans que des preuves expérimentales concluantes aient été données.

En 1938, Aten et Hevesy [2] ont employé la technique des isotopes radioactifs en vue d'étudier l'origine des phosphatides du lait. Cette technique fournit un moyen élégant pour approfondir et préciser les données fournies par les autres méthodes.

Reprenant les recherches de ATEN et Hevesy, nous avons employé le radiophosphore comme moyen d'investigation en l'administrant à des animaux en lactation naturelle ou provoquée par l'implantation d' aestrogènes.

Dans une première série de recherches, faites sur la Chèvre en lactation artificielle, nous avons complété les résultats obtenus par Aten et Hevesy sur quelques points qui méritent d'être rappelés.

Le phosphore lipidique du lait ne devient pas radioactif ni après injection d'une faible dose de phosphate disodique marqué, mesurant $32.10^{6}$ coups, ni après une dose 100 fois plus forte $[3,4]$.

Par contre, le phosphore minéral du lait devient rapidement radioactif et le reste pendant les 15 jours d'expérience. Ce phénomène ne se modifie pas après l'administration d'une dose plus forte, seule l'activité spécifique de la fraction phosphorée étant proportionnellement plus élevée.

La fraction caséinique devient radioactive plus tardivement que la fraction minérale et elle perd son activité vers le $7^{\mathrm{e}}$ jour qui suit l'injection. Il en est de même quand la dose d'isotope administrée est plus élevée.

Un fait mérite d'être souligné à l'examen de ce graphique, à savoir la discordance entre la courbe d'élimination du radiophosphore minéral et celle du radiophosphore protéique. On peut rap-

(1) Bulletin Société Ch. biol., 1951, 33, n*9, 1240. 
les 54 heures de l'expérience; chez les rates, une seule traite a été faite avant la sacrification, 24 heures après l'injection.

Les résultats de ces recherches peuvent être résumés de la manière suivante.

\section{Chienne}

Lait. - L'élimination du phosphore radioactif dans le lait présente les mêmes caractéristiques que celles qui ont été observées chez la chèvre en lactation artificielle. Pour la période de 54 heures, l'activité spécifique du phosphore total a la forme d'un plateau, dont le maximum est situé entre la $4^{\mathrm{e}}$ et la $8^{\mathrm{e}}$ heure qui suit l'injection.

Tissu mammaire. - Dans la glande mammaire de l'animal sacrifié 54 heures après l'injection, le lait résiduel a été extrait par pression, puis le tissu a été broyé (Waring blender) dans une solution de $\mathrm{NaCl}$ à $10 \%$. Les protéines solubles donnent une solution jaune claire légèrement opalescente de $p \mathrm{H}=6,83$. Des fractions aliquotes de cette solution ont été traitées de la manière suivante :

a) Les protéines totales ont été précipitées avec 4 volumes d'acide trichloracétique à $15 \%$.

b) Une autre fraction a été traitée par le sulfate d'ammonium à demi-saturation et à saturation, en vue de séparer les albumines et les globulines.

c) Enfin, dans la troisième fraction, un précipité a été obtenu par addition d'acide acétique à 1,5\% jusqu'à pH 4,60, point isoélectrique de la caséine.

Les protéines isolées ont été délipidées, purifiées par précipitations successives d'après la méthode de Cherbuliez [7], puis desséchées à 1'alcool-éther. Dans la poudre ainsi obtenue, une fraction a été analysée pour la teneur en azote et phosphore total, ainsi que pour la mesure de la radioactivité. Une autre fraction a été hydrolysée avee 10 volumes de $\mathrm{HCl} 3 \mathrm{~N}$ pendant 16 heures. Dans l'hydroIysat neutralisé l'identification de quelques acides aminés a été faite par chromatographie bi-dimensionnelle sur papier (phénolbutanol) et la détermination de la glycine et de la sérine a été faite d'après la technique d'Alexander [8] et de Desnuelce [9]. Das essais de mesure directe de la radioactivité des taches chromatographiques sont restés sans résultat, sans doute en raison de la trop faible quantité d'isotope isolée.

Le tableau suivant résume les résultats obtenus.

On remarque que la plus grande partie des protéines du tissu mammaire est constituée par des globulines, les albumines n'y entrant que pour moins de $5 \%$. En ee qui concerne les protéines précipitée au point isoélectrique de la caséine $(4,60)$, les données ana- 
TABLEAU I

DONNEES ANALYTIQUES CONCERNANT LES DIVERSES FRACTIONS OBTENUES DANS L'EXTRAIT DE GLANDE MAMMAIRE EN LACTATION (rate)

\begin{tabular}{|c|c|c|c|c|}
\hline & $\begin{array}{c}\mathrm{CCl}^{3} \mathrm{COOH} \\
15 \%\end{array}$ & $\begin{array}{l}\mathrm{SO}^{4} \mathrm{Am}^{2} \\
\text { à } 1 / 2 \text { sat. }\end{array}$ & $\begin{array}{l}\mathrm{SO}^{4} \mathrm{Am}^{2} \\
\text { à sat. }\end{array}$ & $\begin{array}{c}\mathrm{CH}^{3} \mathrm{COOH} \\
1,5 \%\end{array}$ \\
\hline $\mathrm{N}$ total $\mu \mathrm{g} / \mathrm{m}$ & 107,2 & 152,4 & 153,0 & 109,2 \\
\hline $\mathrm{N}$ sérine $\mu \mathrm{g} / \mathrm{mg} \ldots \ldots \ldots \ldots$ & 2,0 & 0,84 & - & 3,92 \\
\hline p. 100 de Nl total ......... & 1,87 & 0,55 & & 3,58 \\
\hline$P$ total $\mu \mathrm{g} / \mathrm{mg} \ldots \ldots \ldots \ldots$ & 3,12 & 0,5 & - & 36,2 \\
\hline p. $100 \mathrm{du}$ P. total $\ldots \ldots \ldots \ldots$ & 0,31 & - & & 3,62 \\
\hline P $32 /$ P $31 \mathrm{e} / \mu \mathrm{g} \quad \ldots \ldots \ldots \ldots$ & 44,6 & - & - & 38,4 \\
\hline $\mathrm{P} / \mathrm{N}$ sérine $\ldots \ldots \ldots \ldots \ldots$ & 1,56 & - & - & 9,24 \\
\hline
\end{tabular}

lytiques comparatives de ces deux protéines montrent qu'il existe des différences appréciables, surtout en ce qui concerne le taux phosphoré :

\section{TABLEAU II}

COMPARAISON ENTRE LES DONNEEES ANALYTIQUES DE LA PROTÉINE PHOSPHORÉE MAMMAIRE ET DE LA CASÉINE

\begin{tabular}{|c|c|c|}
\hline & Caséine & $\begin{array}{c}\text { Phosphoprotéine } \\
\text { mammaire }\end{array}$ \\
\hline $\mathrm{N}$ total p. $100 \quad \ldots \ldots \ldots \ldots \ldots \ldots$ & 15,75 & 10,92 \\
\hline P totalp. $100 \ldots \ldots \ldots \ldots \ldots \ldots$ & 0,80 & 3,62 \\
\hline $\mathrm{N}$ sérine $\mathrm{p}, 100 \quad \ldots \ldots \ldots \ldots \ldots \ldots$ & 2,60 & 3,58 \\
\hline $\mathrm{P} / \mathrm{N}$ sérine $\ldots \ldots \ldots \ldots \ldots \ldots \ldots \ldots \ldots$ & 2,21 & 1,56 \\
\hline P libre après fixation sérinique p. 100 & 0 & 74 \\
\hline Point isoélectrique ............ & 4,60 & 4,60 \\
\hline
\end{tabular}

Cette protéine est plus riche en phosphore que la caséine: $3,62 \%$ au lieu de $0,80 \%$. Le rapport $\mathrm{P} / \mathrm{N}$ sérinique est de 9,24 dans la protéine mammaire et de 2,21 dans la caséine. Enfin, le rapport $\mathrm{N}$ sérine/ $\mathrm{N}$ total est de 3,58 dans la protéine mammaire et de 2,40 dans la caséine.

Il s'agit done d'une protéine plus riche en sérine et surtout en phosphore (450\% le taux du $\mathrm{P}$ caséinique). Comme le phosphore ne se fixe sur la sérine que jusqu'à $24 \%$ ( $P$ phosphosérine $=0,24)$ de son taux, le reste doit être lié à un autre corps, soit un acide aminé, soit un autre constituant chimique. Il n'est pas vraisemblable qu'il s'agisse de $\mathrm{P}$ minéral fixé sur une fonction aminée, sur le groupement aminé de la lysine dans l'hypothèse de VAN DER BuRgh [10], car l'activité spécifique de ce corps serait beaucoup 
plus forte et les taches chromatographiques de lysine seraient radioactives [11]. Il y a lieu de penser que cette protéine constitue une étape intermédiaire tissulaire entre la molécule de phosphoprotéine élaborée et celle qui est présente dans le lait. L'hypothèse de TURNER et ses collaborateurs cadre assez bien avec cette cons. tatation si l'on admet que la fraction glucidique de ce précurseur est phosphorylée et arrive dans la glande a vec la copule glucidique à l'état de phosphopyruvate qui se transaminerait ultérieurement. En effet, les données analytiques concernant la protéine isolée par ces auteurs montrent qu'il s'agit d'une globuline constituée pour $9 \%$ d'une fraction glucidique (galactose, mannose et glucosamine). La phosphorylation de cette globuline donnerait un taux de $\mathrm{P}$ variant entre 1,5 et $3,2 \%$, suivant la forme de la copule glucidique. Le pourcentage de $3,6 \%$ trouvé dans la protéine mammaire est assez rapproché de ces données.

\section{Rate}

Le tissu mammaire a été broyé dans l'acide trichloracétique à froid, d'après la méthode de SoHmidt et TANNHAUSER, adaptée à l'usage des isotopes radioactifs [12]. La même technique a été appliquée à une fraction de glande mammaire de chienne en lactation.

Différentes fractions phosphorées ont été déterminées : $\mathrm{P}$ total, $P$ acidosoluble, $P$ minéral, $P$. lipidique, $P$. protéique, $P$ désoxyribonucléique et $P$ ribonucléique. En même temps, la mesure de l'activité de ces fractions a été faite, en précipitant le phosphore minéral de chaque fraction à l'état de phosphate ammoniaco-magnésien, afin d'éviter des erreurs dues à la self-absorption.

Les résultats obtenus sont résumés dans le tableau III.

TABLEAU III

CCNSTITUANTS PHOSPHORES DE LA GLANCE MAMMAIRE DE LA CHIENNE ET DE LA RATE EN LACTATION

\begin{tabular}{|c|c|c|c|c|}
\hline \multirow{2}{*}{ Constituant } & \multicolumn{2}{|c|}{ Chienne } & \multicolumn{2}{|c|}{ Rate } \\
\hline & P 31 & $\begin{array}{c}\text { Activité } \\
\text { spécifique }\end{array}$ & P. 31 & $\begin{array}{l}\text { Activité } \\
\text { spécifique }\end{array}$ \\
\hline$P$ total $\ldots \ldots \ldots \ldots \ldots$. & 1,600 & 16,2 & 1,900 & 56,2 \\
\hline$P$ acide-soluble $\ldots . \ldots \ldots \ldots \ldots$ & 995 & 24,1 & 1,260 & 65,1 \\
\hline $\mathbf{P}$ minéral $\quad \ldots \ldots \ldots \ldots \ldots \ldots$ & 475 & 25,2 & 830 & 81,1 \\
\hline Plipidique $\ldots \ldots \ldots \ldots \ldots \ldots$ & 96 & 10,1 & 210 & 8,3 \\
\hline $\mathbf{P}$ protéique $\ldots \ldots \ldots \ldots \ldots \ldots$ & 625 & (1) 2,6 & 120 & 44,2 \\
\hline P désoxy-ribonucléique ....... & 80 & 14,1 & 235 & 6,5 \\
\hline P ribonucléique .............. & 205 & 22,7 & 310 & 45,5 \\
\hline
\end{tabular}


En comparant les chiffres obtenus à l'analyse du tissu mammaire de la rate avec ceux du tissu mammaire de la chienne, on remarque que :

En dehors de la forte proportion de phosphore protéique dans le tissu mammaire de la chienne en lactation $(31,2 \%)$, par rapport au taux trouvé dans la glande mammaire de la rate $(6,2 \%)$ le reste des constituants phosphorés présente approximativement les mêmes valeurs, l'excès de $P$ protéique diminuant la proportion de $\mathrm{P}$ acido-soluble $(49,8 \%$ chez la chienne par rapport à $71,2 \%$ chez la rate).

L'activité spécifique des fractions phosphorées chez la rate permet quelques remarques intéressantes [13] :

Bien que la concentration en phosphore stable soit presque identique dans les constituants nucléoprotéiques du tissu mammaire, leurs activités spécifiques diffèrent sensiblement, eelle de l'acide désoxyribonucléique étant dix fois plus faible que celle de l'acide ribonucléique, laquelle est du même ordre de grandeur que celle des autres constituants phosphorés analysés.

Il est permis de rapporter cette différence d'activités spécifiques à une différence de vitesse d'intégration du phosphore minéral dans les constituants phosphorés du noyau et du cytoplasme sécrétoire. En effet, une activité spécifique plus faible dans les constituants chimiques du noyau plaide en faveur d'une stabilité plus grande des constituants phosphorés nucléaires et respectivement pour une circulation (turnover) accélérée des constituants phosphorés cytoplasmiques. Il s'ensuit que le eytoplasme a une participation prépondérante dans le mécanisme de la sécrétion lactée, l'excrétion du lait se faisant comme un tout, dès qu'une ou plusieurs "substances pilotes " de la sécrétion atteignent un rapport limite. Parmi ces corps capables de diriger l'excrétion du lait élaboré, il est probable que les nucléoprotéines cellulaires ont une importance prépondérante.

La différence des activités spécifiques constatée chez la chienne peut s'expliquer par la différence dans le moment du prélèvement qui pourrait correspondre à une autre phase sécrétoire,

Les résultats expérimentaux exposés peuvent être résumés de la manière suivante :

L'emploi du radiophosphore minéral a permis de préviser le fait. que le lait élimine une fraction n'excédant pas $25 \%$ du radiophosphore injecté sous forme de phosphore protéique. $\Pi$ y a une différence dans l'élimination du phosphore protéique par rapport à celle du P minéral, indépendamment de la dose d'isotope administrée. L'analyse de cette différence conduit à la conclusion que le phosphore minéral injecté s'intègre dans un précurseur formé en 
quantité limitée, résultant des conditions expérimentales; c'est le précurseur qui se transforme ultérieurement en caséine. Il est permis de penser que ce précurseur est l'acide phospho-pyruvique qui se transforme en phosphosérine. Des recherches sur les constituants protéiques de la glande mammaire en lactation montrent qu'elle contient une protéine plus riche en phosphore qui peut être considérée comme un autre chaînon de ce précurseur, apparenté à la glycoprotéine de TURNer, probablement phosphorylée.

Le mécanisme de transformation de ce précurseur en caséine, ainsi que l'excrétion de la molécule de caséine une fois élaborée paraît dépendre étroitement des constituants nucléo-protéides nucléaires et plasmatiques. Le phosphore désoxyribonucléique étant plus stable que le phosphore ribonucléique cytoplasmique, ce fait autorise l'hypothèse que l'excrétion de la caséine se fait d'emblée avec les autres constituants, quand un certain rapport nucléo-plasmatique est atteint.

(Laboratoire de Physiologie. Institut National Agronomique, Paris. Institut de Microbiologie et d'Hygiène, Université de Montréal, Montréal.)

\section{BIBLIOGRAPHIE}

[1] E. P. Reineke, M. B. Williamson et C. W. Turner. - Journal Biol. Chem., 1941, 138, 83.

[2] A. H. W. Amen et G. Hevesy. Nature, 1938, 142, 111.

[3] H. Stmonnet, P. Bonet-Maury et J. Sternberg. C. R. Acad. Sc., $1950,230$.

[4] P. Bonet-Maury, G. Demaux, H. Simonnet et J. Sternberg. Résultats non publiés.

[5] M. Вуснкоw. Exposés annuels de Bioch. médicale, 1949, 10, 226.

[6] J. Colas, H. Le Bars, H. Simonnet et J. Sterngerg. Ann. Inst. nat. Agron., 1950, 37, 5.

[7] E. Cherbuliez et P. Baudet. Helv. Chimica Acta, 1950, 33, 398.

[8] B. Alexander et G. Seligman. Journ. Biol. Chem., 1945, $159,9175$.

[9] P. Desnuelie, S. Antonin et M. Naudet. Travaux membres Soc. Chim. biol., 1944, 26, 1168.

[10] P. Van der Burgh. Nethert. Milk Dairy J., 1947, 1, 69.

[11] H. Le Bars, H. Simonnet et J. Sternberg. C. R. Acad. Sci., 1951, 232, 1148 .

12] J. A. McCarter et E. Steljes. Can. J. Med. Res., 1948, 26 E, 333.

[13] J. Stem rnerg. C. R. Acad. Sci., 1950, $230,2341$. 